\title{
Tracing Black Hole Signatures in Several Black Hole Candidates Based on Their X-Ray Spectral Evolution
}

\author{
Fahmi Iman Alfarizki ${ }^{1, *}$ and Kiki Vierdayanti ${ }^{2}$ \\ ${ }^{1}$ Astronomy Study Program, Faculty of Mathematics and Natural Sciences, Institut Teknologi \\ Bandung, Indonesia \\ ${ }^{2}$ Astronomy Research Division, Faculty of Mathematics and Natural Sciences, Institut Teknologi \\ Bandung, Indonesia
}

\begin{abstract}
Investigation of spectral evolution of four black hole candidates was carried out by using color-color diagram as well as spectral fitting on Swift/XRT data. Newly found candidates, which are classified as low-mass X-ray binary system based on their transient nature, are the focus of our work. We compare their spectral evolutions to that of XTE J1752-223, a transient system and a more convincing black hole candidate whose mass has been determined from spectral-timing correlation scaling. In addition, comparison to Cygnus X-1, a well-known stellar-mass black hole, was done despite its persistent nature. The spectral fitting, by using a combination of thermal disk and non-thermal component model, results in the innermost temperature values in the range of the typical innermost temperature of black hole binary which is $0.7-1.5 \mathrm{keV}$. The spectral evolutions of the candidates bear a resemblance to both Cygnus X-1 and XTE J1752-223. We note that during Swift/XRT observations, the spectra of Cygnus X-1 and IGR J17451-3022 are mostly dominated by the nonthermal component. We conclude that the compact object of MAXI J1535571 and MAXI J1828-249 is highly likely to be a black hole. However, the lack of data rendered conclusive result impossible for IGR J17454-2919.
\end{abstract}

Keywords: black hole binary, black hole candidates, black hole signatures, low-mass X-ray binary, spectral evolution.

\section{Introduction}

Direct evidence of astrophysical black hole remains difficult to obtain even after the first image of black hole's shadow, which happened to be a supermassive black hole in the center of M87 [1], has been obtained by Event Horizon Telescope (EHT) team. The difficulties are more pronounced in the case of stellar-mass black holes due to their much smaller dimension. On the other hand, their extremely compact dimension give rise to much better opportunities to study their variability. Indirect evidence, such as dynamical mass

*Corresponding author: fahmimn21@students.itb.ac.id 
estimation in some X-ray binary systems, has been used to show the existence of stellarmass black hole in our Galaxy and other nearby galaxies (e.g. [2-3]). However, this method cannot always be applied to X-ray binaries, especially transient systems with extremely dim optical counterpart.

Fortunately, radiation from the accretion disk surrounding a black hole in X-ray binary systems can easily be detected and it may reveal information from the innermost region near the black hole. Many well-known stellar-mass black hole candidates are discovered in $\mathrm{X}$-ray binaries. It is important to note that distinguishing a stellar-mass black hole from a neutron star is not trivial due to similar relativistic effects [4]. However, since a neutron star has a solid surface while a black hole does not, we expect different accretion properties closed to the compact object. A black hole has the innermost stable circular orbit (ISCO) radius which is usually assumed as the location of the innermost radius of the accretion disk in thermal dominant state. However, extensive study of black hole binaries in our Galaxy has revealed that these sources exhibit spectral variability [5]. Done \& Gierlinski (2003) have shown that spectral evolution of black hole can be distinguished from that of neutron star in X-ray binary systems. We utilized the method used in Done \& Gierlinski (2003) to trace black hole signature in four newly found black hole candidates. We present our data and methods in section 2. The results of our study are presented in section 3. Section 4 is dedicated for discussion while section 5 concludes our study.

\section{Data and Methods}

\subsection{Data Selection and Reduction}

Our study focus on black hole candidates in transient systems. The list of the candidates was obtained from an on-line catalogue of stellar-mass black holes in X-ray binaries [6]. The catalogue consists of 60 stellar-mass black hole candidates and it is still growing. We chose four newly found candidates which are MAXI J1535-571, MAXI J1828-249, IGR J17451-3022, and IGR J17454-2919. The list of the candidates are presented in Table 1. Those candidates will be compared to XTE J1752-223, a more convincing candidate of black hole binary, whose mass has been determined from spectral-timing correlation scaling [7]. Also, we compared all candidates to Cyg X-1, a well-known black hole binary, despite its persistent nature.

Table 1. The comparison of amount of data, observation period, and count rates range of black hole candidates with Cyg X-1.

\begin{tabular}{|l|r|r|r|}
\hline \multicolumn{1}{|c|}{ Objects } & \multicolumn{1}{|c|}{$\begin{array}{c}\text { Number of } \\
\text { Data }\end{array}$} & $\begin{array}{c}\text { Observations Time Range } \\
\text { (MM/YYYY) }\end{array}$ & $\begin{array}{c}\text { Count Rates Range } \\
\text { (counts/s) }\end{array}$ \\
\hline Cyg X-1 & 113 & $12 / 2004-03 / 2019$ & $4.09-914.6$ \\
\hline XTE J1752-223 & 66 & $09 / 2009-07 / 2010$ & $3.95 \times 10^{-4}-329.1$ \\
\hline MAXI J1535-223 & 109 & $09 / 2017-06 / 2019$ & $0.07-902.3$ \\
\hline MAXI J1828-249 & 48 & $10 / 2013-04 / 2014$ & $3.31 \times 10^{-4}-205.1$ \\
\hline IGR J17451-3022 & 41 & $09 / 2012-05 / 2015$ & $3.37 \times 10^{-3}-2.45$ \\
\hline IGR J17454-2919 & 25 & $052007-09 / 2016$ & $9.08 \times 10^{-4}-1.72$ \\
\hline
\end{tabular}

Most of data are in windowed-timing mode since the candidates are bright Galactic Xray sources. We used Heasoft 6.26.1 for data processing and Xspec 12.10.1f for spectral fitting. We did pile-up correction for count rate greater than $\sim 100 \mathrm{ct} / \mathrm{s}$ and $\sim 0.5 \mathrm{ct} / \mathrm{s}$ for 
windowed timing mode and photon counting mode, respectively. Each data is grouped so that to have a minimum 20 counts per bin.

\subsection{Spectral Fitting}

We only used a combination of simple models to fit each spectra. The thermal component is modelled with multi-color disk spectrum, namely diskbb in Xspec, and the non-thermal component is modelled with power-law spectrum, namely powerlaw in Xspec. The Tübingen-Boulder ISM absorption model is also incorporated in the fitting. In our study, the chi-squared statistics can be used due to high values of total counts as well as count rate (as can be seen in Table 1) in most data. Since not all fittings result in acceptable values of reduced chi-squared, for further analysis, we only used data with $\chi^{2} / v<2$.

\subsection{Spectral Evolution Analysis}

To get an overview of source behaviour, we used color-color and hardness-intensity diagrams. Since the count rate detected within a certain energy range depend on both the instrument response and on the observing column towards the source, we used unabsorbed flux over a given energy band to get a measure of the true source behaviour on the diagrams.

For the color-color diagram, we used three energy bands to form the soft and hard colors, $0.6-2.5 \mathrm{keV}, 2.5-4.5 \mathrm{keV}$, and $4.5-10 \mathrm{keV}$. Following Reynolds \& Miller (2013), the soft and hard colors are defined as the ratio of unabsorbed fluxes in the $2.5-4.5$ $\mathrm{keV}$ and $0.6-2.5 \mathrm{keV}$ bands and in the $4.5-10 \mathrm{keV}$ and $2.5-4.5 \mathrm{keV}$ bands, respectively. Assuming that the relative uncertainty of the unabsorbed flux is comparable to that of the count rate, we could estimate the uncertainty of the unabsorbed flux.

For the hardness - intensity diagram, the intensity was obtained from the calculation of unabsorbed fluxes in the $0.3-10 \mathrm{keV}$ bands. As for the hardness, we defined hardness ratio as the ratio of unabsorbed fluxes in the $1.5-10 \mathrm{keV}$ and $0.3-1.5 \mathrm{keV}$ bands, as normally used in Swift/XRT data.

\section{Results}

\subsection{Spectral fitting}

Figure 1 shows the histogram of reduced chi-squared values of each candidate. We found that most of data have reduced chi-squared less than 2, although there are several data whose reduced chi-squared close to zero, which normally happens to spectra with large uncertainty. Hence, we only used data which result in reduced chi-squared between $0.5-$ 2.0 for further analysis. We found that the majority of data of each candidate reveal that the innermost temperatures are in the range of black hole binary, which is between 0.5 to 1.5 $\mathrm{keV}$. Despite some of them are higher or lower than that range (See Figure 2). The photon index of power-law for all candidates are very high and some of them have a wide range of photon index such as MAXI J1535-571, IGR J17451-3022, and MAXI J1828-249 as shown in Figure 3. 

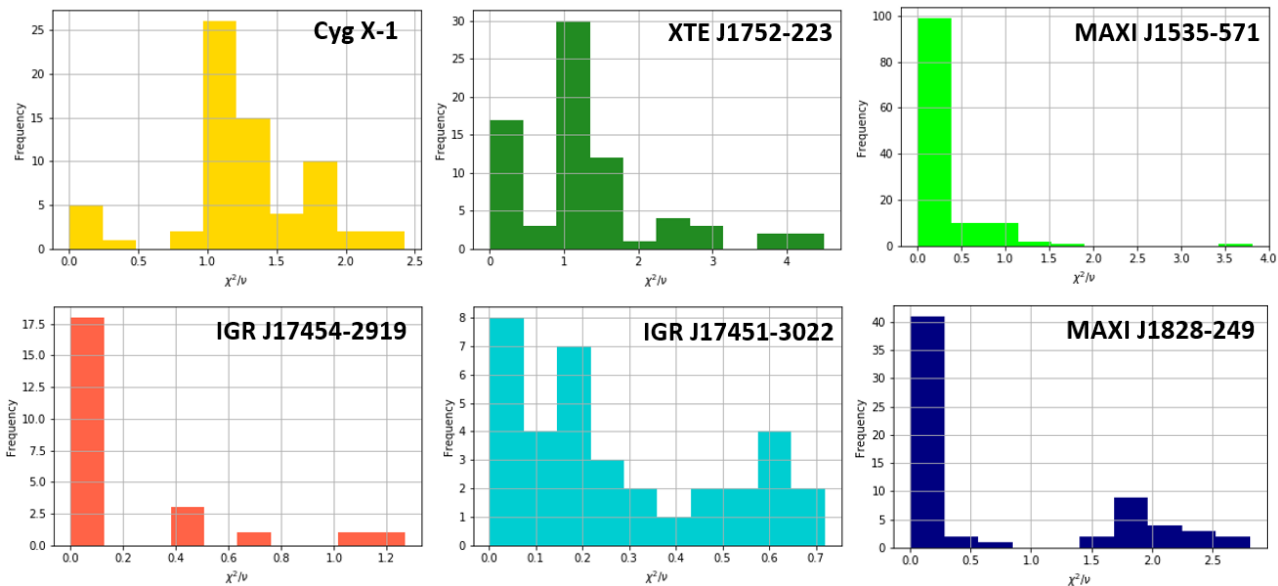

Fig. 1. Distribution of reduced chi-squared values of each candidate.
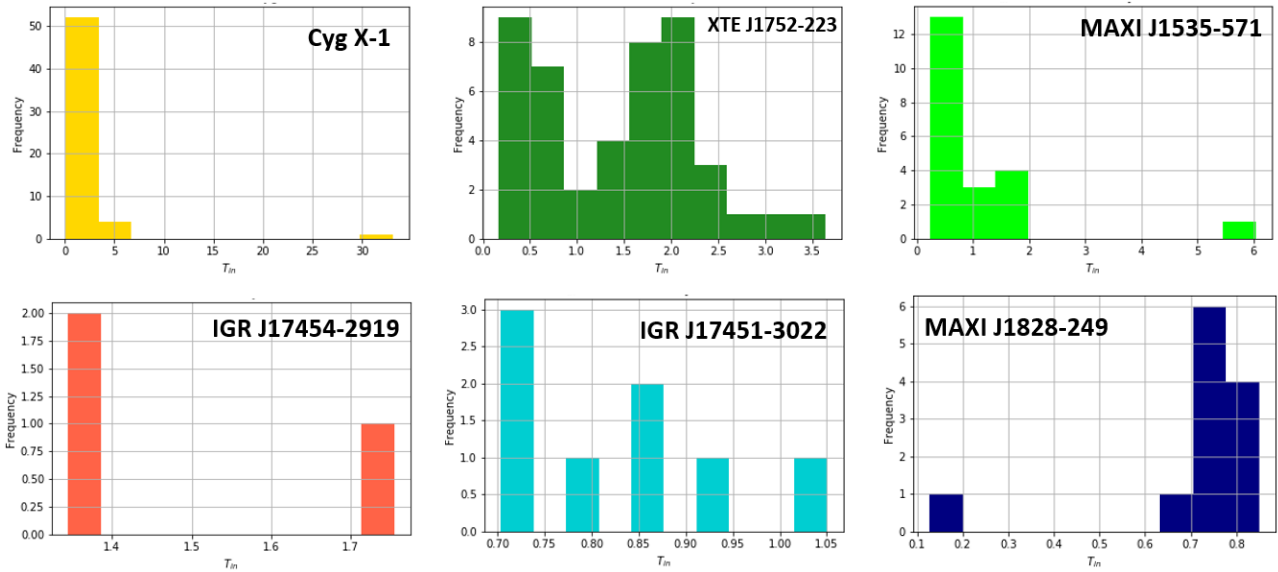

Fig. 2. Distribution of the disk innermost temperature for each candidate.
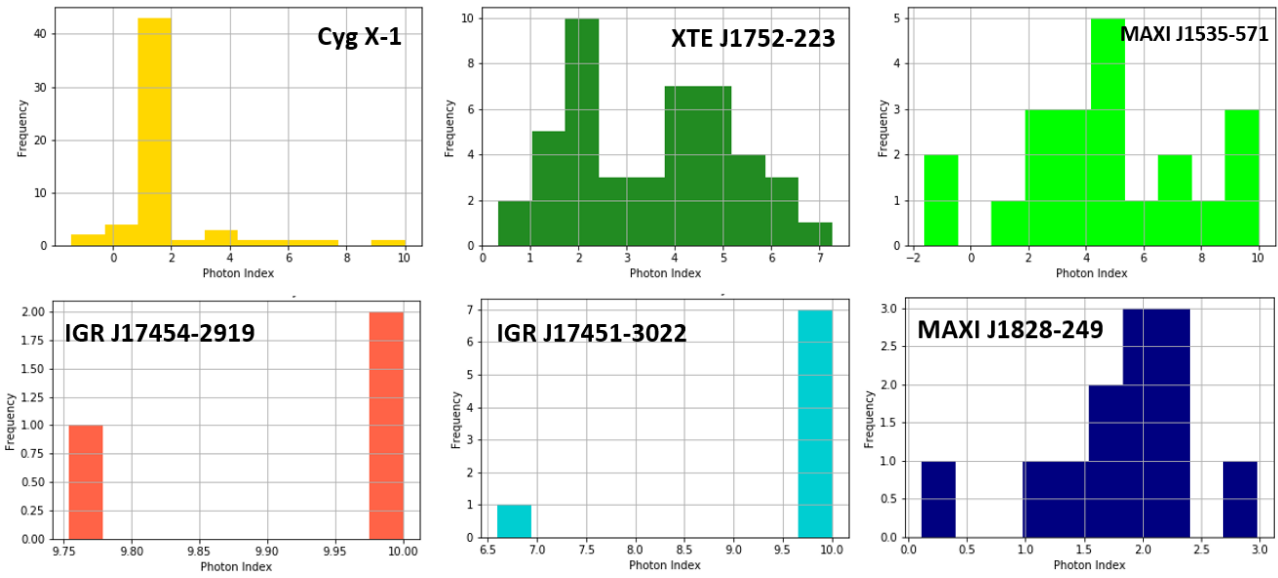

Fig. 3. Distribution of the photon index of power-law for each candidate. 

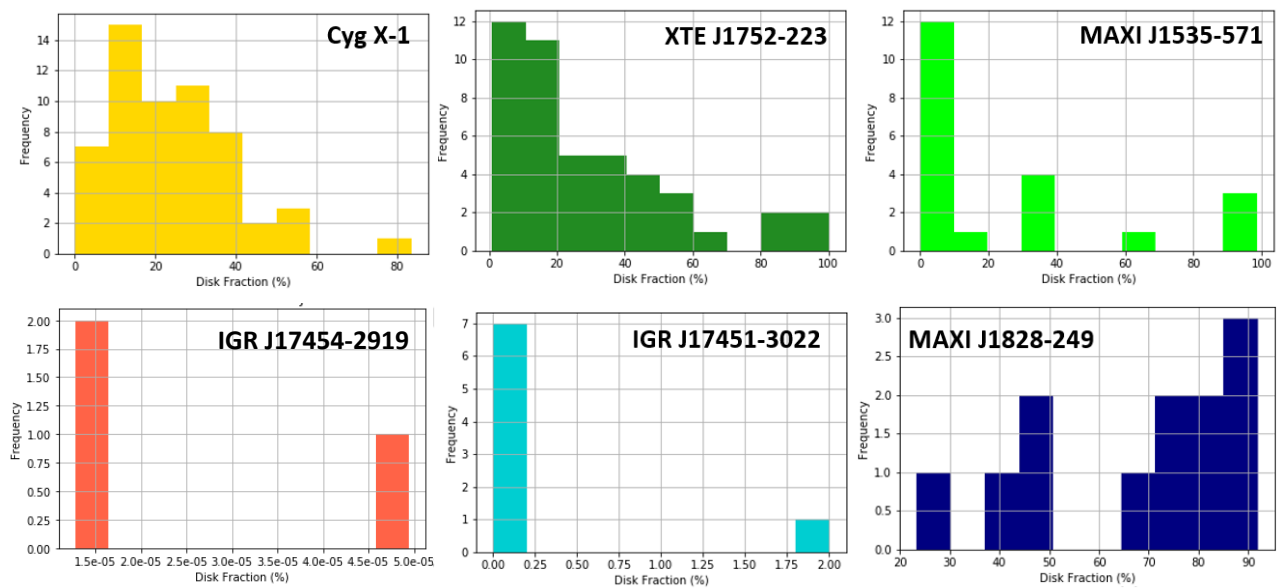

Fig. 4. Distribution of disk component flux to total flux ratio of each candidate.

We also measured the ratio between the disk component unabsorbed flux and total unabsorbed flux. Figure 4 shows that majority of data are not in thermal dominated state in which the disk contribution to the spectra must be greater than 75\% [5]. Only MAXI J1828249 has most data in thermal dominant state. From this result, we expect that Cyg X-1, IGR J17454-2919, and IGR J17451-3022 do not reveal the evidence of ISCO due to lack of data in thermal dominant state.

\subsection{Spectral Evolution}

Figure 5a shows the color-color diagram of Cyg X-1 and XTE J1752-223, while Figure $5 \mathrm{~b}$ shows the color-color diagram of the candidates. The orange solid lines in both panels represents the powerlaw model. The blue solid lines in both panels represents the diskbb model. The spectral evolution of Cyg X-1 fall on the powerlaw model track which consistent to the fact that Cyg X-1 spends most of the time in hard state (e.g. [8,9]). The spectral evolution of XTE J1752-223 varies from hard state to ultrasoft state. Several spectra of Cyg X-1 and XTE J1752-223 occupy an area in soft color between 0.2 to 0.6 and not all candidates occupy that range. Only MAXI objects do. Others, however, are very soft. These spectral evolutions resemble those constructed by Done \& Gierlinski (2003) for a sample of black holes observed by $R X T E$.

We built the light curves and hardness ratio evolution in Figure 6 to look at the transient behaviour. Cygnus X-1 reveals a persistent nature and spends most of the time in a hard state as can be confirmed by the color-color diagram. We found an outburst feature like in XTE and both MAXI objects. While in both IGR objects, the range of count rate is too narrow for a typical outburst episode of transient black hole binary. We can also see that the hardness ratio of both IGR objects are very low, implying that the thermal component predominates the spectra. Also, there is no type I burst discovered on the light curves of all candidates. 


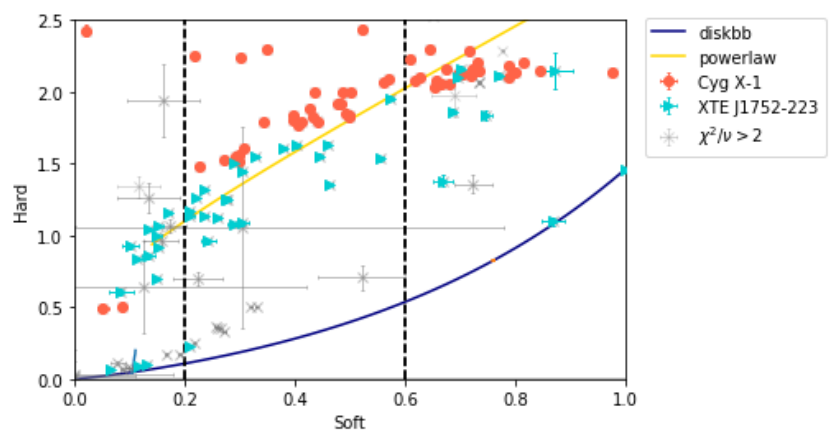

(a)

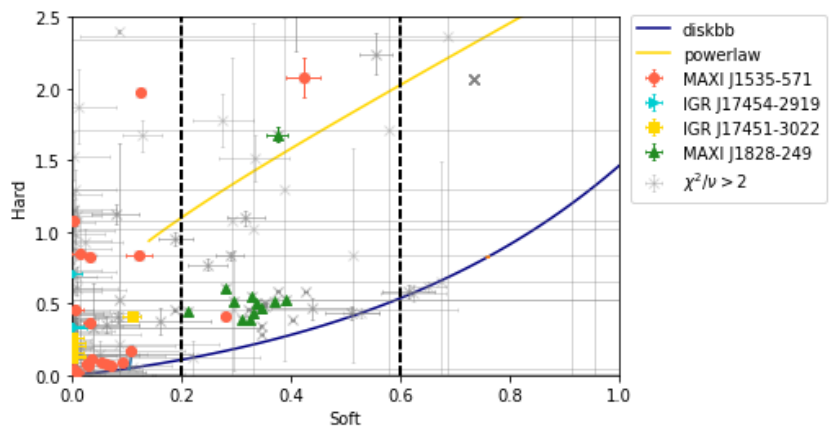

(b)

Fig. 5. Color-color diagram of Cyg X-1 and XTE J1752-223 (a) and all candidates (b). The dashed black lines are soft color which correspond to $0.7-1.5 \mathrm{keV}$ of the disk innermost temperature.

In the hardness-intensity diagram as shown in Figure 7, we can trace the black hole spectral evolution. Cyg X-1 and XTE 1752-223 show Q-shaped pattern on the spectral evolution track. These consistent to black hole binary evolution. When the luminosity is high, then soft component is dominant, and vice versa. Resemblance to the spectral evolution track to CygX-1 and XTE 1752-223 can only be seen in MAXI 1535-571. The spectral evolution track of the rest of the candidates is inconclusive due to the lack of data.

\section{Discussion}

The area in soft color between 0.2 to 0.6 in the color-color diagrams in Figure $5 \mathrm{~b}$ can not be occupied by neutron stars because the neutron stars have a additional flux from their surface [8]. Hence, MAXI J1828-249 behavior in color-color diagram is in a good agreement with that of black hole. For the rest of the candidates, however, we cannot, based on the color-color diagram, see their resemblance to that of black hole. Their extremely soft color indicate resamblence to that of super-soft source which are known to host a white dwarf. 

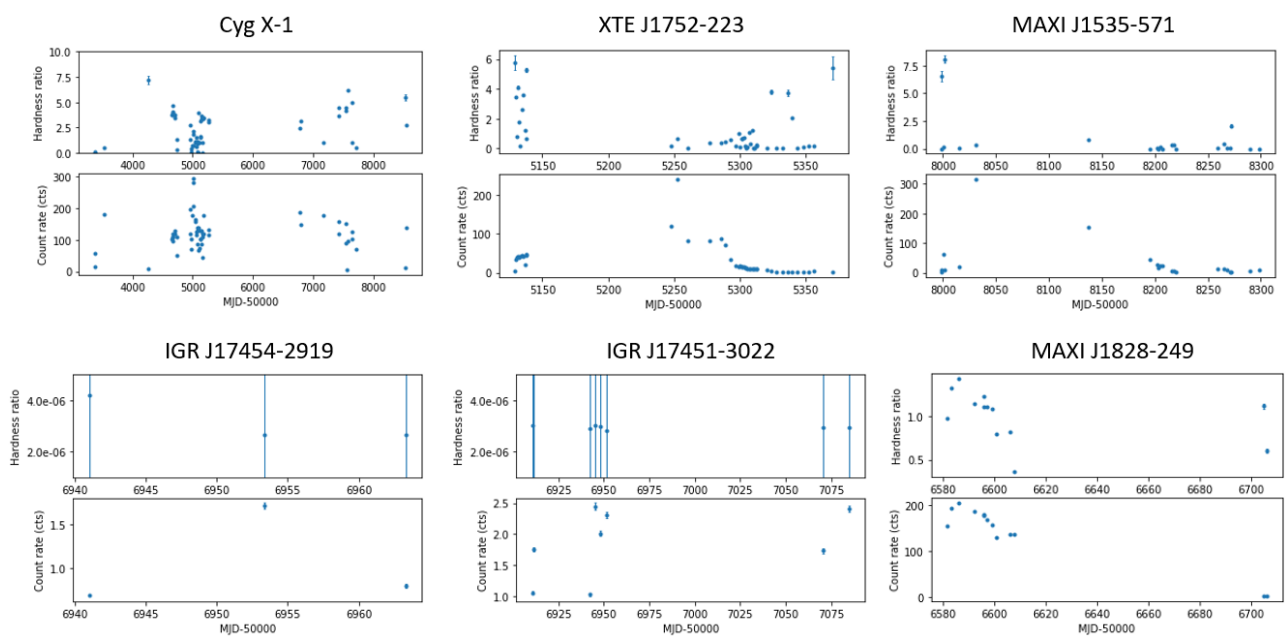

Fig. 6. The upper panel is the hardness ratio evolution and the lower panel is the light curve in terms of count rate.
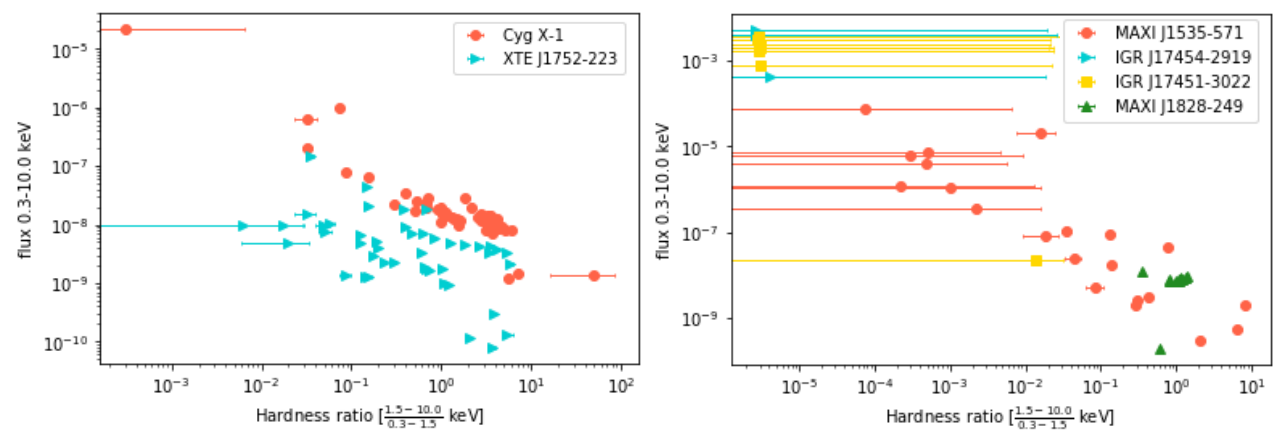

Fig. 7. Hardness-intensity diagrams of Cyg X-1 and XTE J1752-23 (left-hand panel) and the candidates (right-hand panel).

Figure 7 reveal that MAXI J1535-571 clearly follow the spectral evolution tracks of Cyg X-1 and XTE J1752-223. Although MAXI J1828-249, IGR J17454-2919, and IGR J17451-3022 do not show full spectral evolution tracks, we cannot rule out the possibility that MAXI J1828-249 and both IGR objects follow the spectral evolution track of Cyg X-1 and XTE J1752-223. Another evidence for the black hole event horizon can be obtained from the values of the innermost radius of accretion disk. However, it is necessary to check the relation between the innermost disk temperature and the luminosity to make sure that the spectra are in thermal dominated state.

In thermal dominated state, the innermost radius of the accretion disk can be assumed to reach the ISCO radius. Moreover, we expect that the disk luminosity and the innermost temperature of the disk obey $L \propto T^{4}$. Since we do not have the distance parameter, we measured the disk luminosity in term $L / d^{2}$. Further, we also expect that the innermost radii of accretion disk remain constant and can be assumed to coincide with the ISCO radius. 


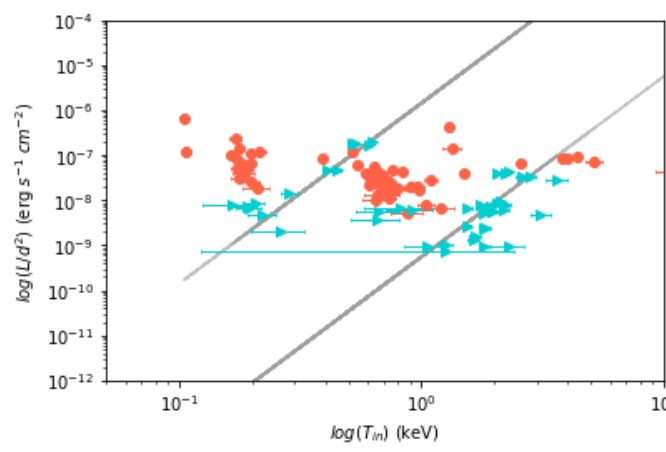

4. Cyg X-1

+ XTE J1752-223

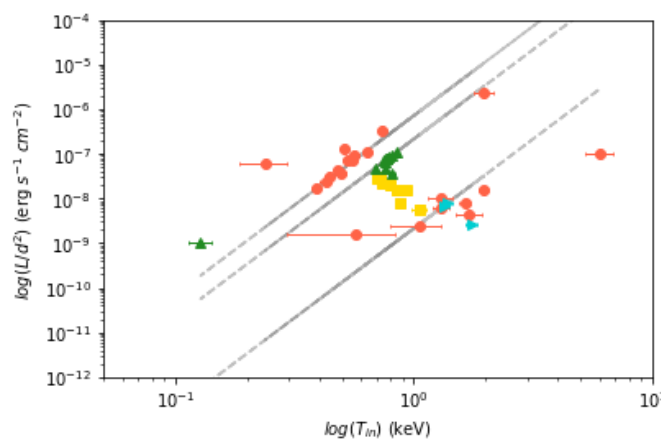

- MAXI J1535-571

IGR J17454-2919

IGR J17451-3022

* MAXIJ1828-249

Fig. 8. The innermost disk temperature and luminosity relations. The lines are the $L / d^{2}$ as a function of $T^{4}$.

Figure 8 shows the relation between the innermost disk temperature and luminosity of Cyg X-1 and XTE J1752-223 (upper panel) and all candidates (lower panel). Some spectra of XTE J1752-223 obey $L \propto T^{4}$ relation and we can expect that this black hole shows the evidence of ISCO and thus the evidence of black hole event horizon. Cyg X-1 clearly does not obey $L \propto T^{4}$ relation since most of time it is in the hard state. The persistent nature of Cyg X-1 can be seen in this diagram in which the values of $L / d^{2}$ remain high and constant. Both MAXI objects obey $L \propto T^{4}$ relation in which we can expect that the spectra are in thermal dominated state. Moreover, MAXI objects also reveal the existence of ISCO in the luminosity-radius diagram (Figure 9). IGR J17451-3022 does not obey the relations and this result consistent to the fact that non-thermal component is dominant in all spectra of IGR J17451-3022 as shown in Figure 4. Some data of IGR J17454-2919 fall into the line of $L \sim T^{4}$ relation, but we cannot conclude that the spectra are in thermal dominated state. 

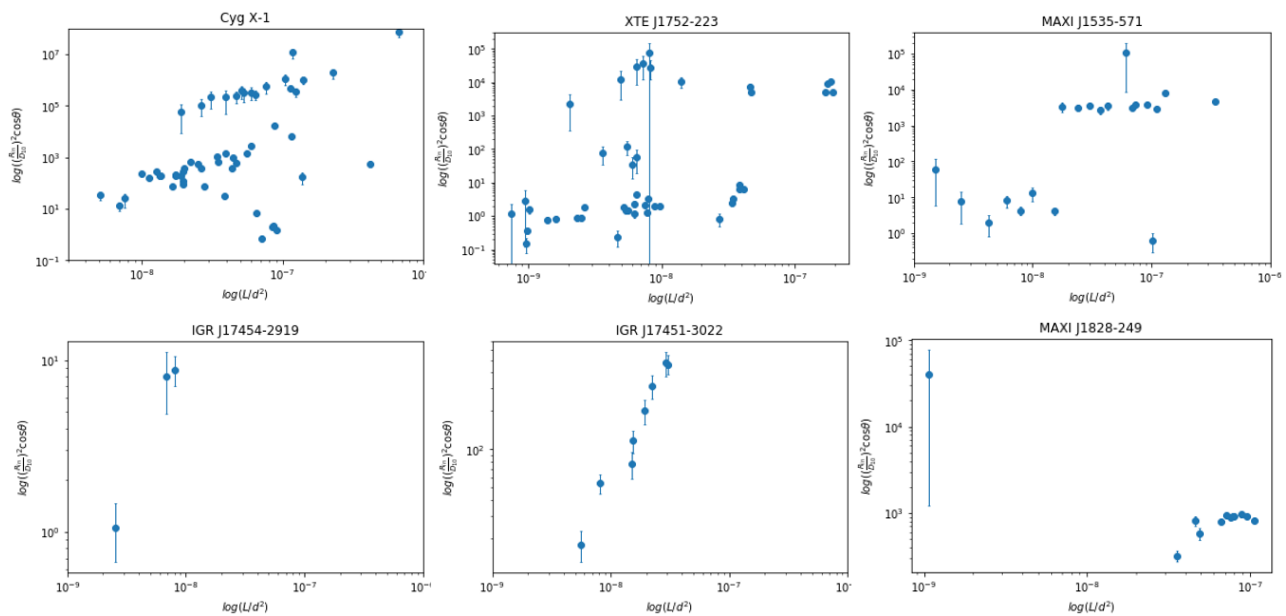

Fig. 9. The luminosity and the innermost radius relations. Since we do not have the distance and the inclination of the disk, we used normalization of diskbb which is correlated to $\left(R_{\text {in }} / D_{10}\right)^{2} \cos \theta$.

As we expected in previous discussion, XTE J1752-223, MAXI J1535-571, and MAXI J1828-249 reveal the existence of the ISCO in some spectra in which the innermost radius of accretion disk remain constant (See Figure 9). However, we did not discover the existence of the ISCO in Cyg X-1 and IGR J17451-3022. This result is consistent to the fact that Cyg X-1 and IGR J17451-3022 are mostly in hard state. Unfortunately, we cannot find any evidence of black hole for IGR J17454-2919, due mostly to the lack of data.

\section{Conclusion}

Spectral evolution of the black hole candidates has been investigated and compared to those of Cyg X-1 and XTE J1752-223. The spectral evolution of MAXI J1535-571 is very similar to those of Cyg X-1 and XTE J1752-223. Although MAXI J1828-249, IGR J174542919, and IGR J17451-3022 do not show full spectral evolution tracks, we cannot rule out the possibility that MAXI J1828-249 and both IGR objects follow the spectral evolution track of Cyg X-1 and XTE J1752-223. MAXI J1535-571 and MAXI J1828-249 reveal an evidence of ISCO radius which is the most prominent black hole signatures. Innermost disk radii of IGR J17451-3022 do not remain constant because the spectral evolutions are not in thermal state. Nevertheless, IGR J17451-3022 reveals several evidences and it is more likely to be a black hole. Because lack of data, IGR J17454-2919 does not reveal the evidence of ISCO black hole. However, it reveals some signatures of black holes and must be further examined.

\section{References}

1. The EHT Collaboration et al., Astrophys. J. Lett., First M87 Event Horizon Telescope Results. I. The Shadow of the Supermassive Black Hole, 875, L1 (2019)

2. A. H. Prestwich, R. Kilgard, P. A. Crowther, S. Carpano, A. M. T. Pollock, A. Zezas, S. H. Saar, T. P. Roberts and M. J. Ward, Astrophys. J. Lett., The Orbital Period of the Wolf-Rayet Binary IC 10 X-1: Dynamic Evidence that the Compact Object Is a Black Hole, 669, L21-L24 (2007) 
3. D. Mata Sánchez, T. Muñoz-Darias, J. Casares, J. M. Corral-Santana, and T. Shahbaz, Mon. Not. R. Astron. Soc., Swift J1357.2-0933: A Massive Black Hole in the Galactic Thick Disc, 454, 2199-2204 (2015)

4. N. Ramesh, A\&G, Evidence of the Black Hole Event Horizon, 44, 6.22-6.25 (2003)

5. R. A. Remillard and J. E. McClintock, Annu. Rev. Astro. Astrophys., X-Ray Properties of Black-Hole Binaries, 44, 49-92 (2006)

6. J. M. Corral-Santana, J. Casares, T. Muñoz-Darias, F. E. Bauer, I. G. Martínez-Pais, and D. M. Russell, Astron. Astrophys, BlackCAT: A Catalogue of Stellar-mass Black Holes in X-ray Transients, 587, A61 (2016)

7. N. Shaposhnikov, C. Markwardt, J. Swank and H. Krimm, Astrophys. J., Discovery and Monitoring of a New Black Hole Candidate XTE J1752-223 with RXTE: Rms Spectrum Evolution, Black Hole Mass, and the Source Distance, 723, 1817-1824 (2010)

8. C. Done and M. Gierlinski, Mon. Not. R. Astron. Soc., Observing the Effects of Event Horizon in Black Holes, 342, 1041-1055 (2003)

9. M. T. Reynolds and J. M. Miller, Astrophys. J., A Swift Survey of Accretion onto Stellar-Mass Black Holes, 769, 16 (2013) 